\title{
Analysis and Simulation of the Pi of the Sky Detector Response
}

\author{
L. W. Piotrowski, A. F. Żarnecki
}

\begin{abstract}
The Pi of the Sky project observes optical flashes of astronomical origin and other light sources variable on short timescales, down to tens of seconds. We search mainly for optical emissions of Gamma Ray Bursts, but also for variable stars, blazars, etc. Precise photometry with a very large field of view $\left(20^{\circ} \times 20^{\circ}\right)$ requires a careful study and modelling of a point spread function (PSF), as presented in this paper.
\end{abstract}

Keywords: Gamma-ray Bursts (GRB), point spread function (PSF), wide-field camera.

\section{Introduction}

The Pi of the Sky experiment [2] is designed for continuous monitoring of a large fraction of the sky with high (in astronomical terms) time resolution $(\sim 10 \mathrm{~s})$. A real time analysis of the data stream, based on a multi-level triggering system, allows discoveries of GRB optical counterparts independent from satellite experiments. Additionally, the self triggering capabilities allow the detection of other rapidly varying sources, such as nova and flare stars or even as yet unclassified phenomena. This approach resulted in autonomous detection of the "naked-eye" burst GRB080319B at its very beginning [3].

To meet the requirement for monitoring a large fraction of the sky, the Pi of the Sky apparatus makes use of cameras with a very wide field of view - about $20^{\circ} \times 20^{\circ}$ each. However, for stars positioned far from the optical axis, this causes significant deformations of images, much bigger than in other astronomical experiments (as in the case of GRB080319B, see Figure 1, left).

Therefore both profile and aperture astrometric and photometric algorithms, which assume standard PSF shape, introduce large uncertainties. To im- prove the brightness and coordinate measurements, an elaboration of PSF in the Pi of the Sky system is required. This requires high-precision profiles of the point-sources in different parts of the frame, and a mathematical model that will describe them properly.

\section{Laboratory measurements and modelling}

The derivation of a PSF requires a star profile with sub-pixel resolution, which can be obtained from a superposition of single star images. However, the superposition of real images introduces big uncertainties due to the fact that the exact shape and thus the centre of the star image is unknown. Additionally, stars are blurred due to mount vibrations when following the sky movement, etc. These factors show that ample data needs to be obtained in laboratory measurements.

The apparatus for the laboratory measurements consisted of a LED diode (colour or white) placed behind a pinhole $0.1 \mathrm{~mm}$ in diameter and placed at a distance of $22 \mathrm{~m}$ from a CCD camera ${ }^{1}$. The pixel size
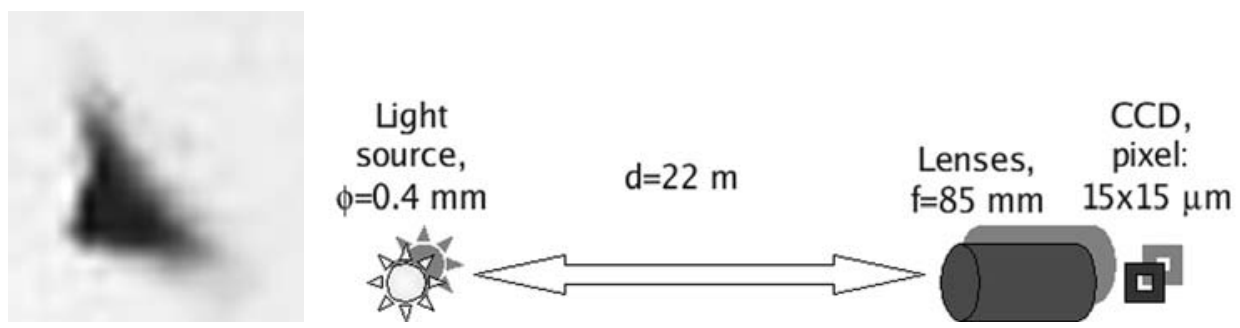

Fig. 1: Left: The second exposure of GRB 080319B taken with the Pi of the Sky apparatus, during which the optical emission was strongest. The GRB was recorded close to the corner of the frame, thus its shape is very deformed; Right: The setup for laboratory measurements

\footnotetext{
${ }^{1}$ The standard $\mathrm{Pi}$ of the Sky camera consists of custom designed electronics with readout noise of $\sim 16 e^{-}$at $2 \mathrm{Mpixels} / \mathrm{s}$, commercial $\sim 2000 \times 2000$ pixel CCD and Canon EF lenses with $f=85 \mathrm{~mm}$ and $f / d=1.2$.
} 
of the CCD sensor was $15 \times 15 \mu \mathrm{m}$ (Figure 1, right). This setup gives a geometrical spot size of the diode on the CCD sensor of less than 0.1 pixel - fulfilling the requirement of a point source.

\section{$2.1 \quad$ Intra-pixel measurements}

The way in which the CCD sensor is designed causes a single-pixel sensitivity to light to be spatially nonuniform [4]. This is mainly due to the electrodes placed across the pixels and channel stops separating the columns of the sensor. This setup with an additional diaphragm $20 \mathrm{~mm}$ in radius on the lenses (reducing the PSF size) enabled measurements of two functions describing the non-uniformity: the pixel response function (PRF) and the pixel sensitivity function.
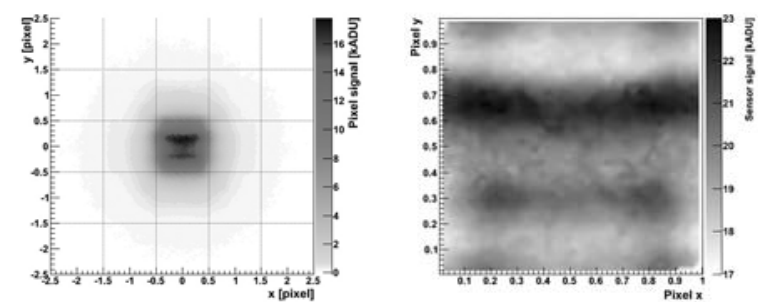

Fig. 2: Left: pixel response function; Right: pixel sensitivity function for a red diode

PRF describes a single pixel signal value vs. the position of the spot relative to the pixel edge. A spot only partially contained in the pixel causes a sudden drop in PRF close to the pixel border (Figure 2, left). However, the function is non-zero for a spot fully outside the pixel. This may be caused by PSF size, by diffraction of the spot, or by a charge diffusion between the pixels. In the last case, the PRF contributes to the whole shape of PSF.

The pixel sensitivity function is defined in a similar way to PRF, but an overall CCD signal value is taken instead of the single pixel signal. Changes in pixel sensitivity are the main factor responsible for the signal changes caused by movement of the image across the CCD. With knowledge of the function and the position of the centre of the source in the pixel one can compensate for this effect, performing a more precise measurement of the brightness.

\subsection{PSF measurements and modelling}

A high resolution profile for selected coordinates in the frame was obtained using multiple images of a diode. Each exposure was taken for a specific position of the centre of the diode and the full set of images covered $10 \times 10$ points inside a single pixel. All the images were superimposed, taking into account the coordinates of each image. Sample profiles obtained for 0, 800 and 1400 pixels from the frame centre along the diagonal are shown in Figure 3.

The spread of the image of the point source, PSF, is caused by deformations of the wavefront of the light passing through the optical system [1]. In the Pi of the Sky case, the PSF should be described by a Rayleigh-Sommerfeld diffraction formula with aberrations, within a Kirchhoff approximation. In the case of the wide-field lenses used in our project, it is not possible to use any of the paraxial approximations that lead to an FFT, thus the formula has to be calculated directly. Therefore, even though this approach should finally lead to satisfactory results, a faster model had to be developed. We use a set of modified Zernike polynomials to describe the image directly. For each measured profile, PSF modelling is obtained by fitting polynomial coefficients to the data (Figure 4). The general model is obtained by interpolating the coefficients determined for selected coordinates on the frame.
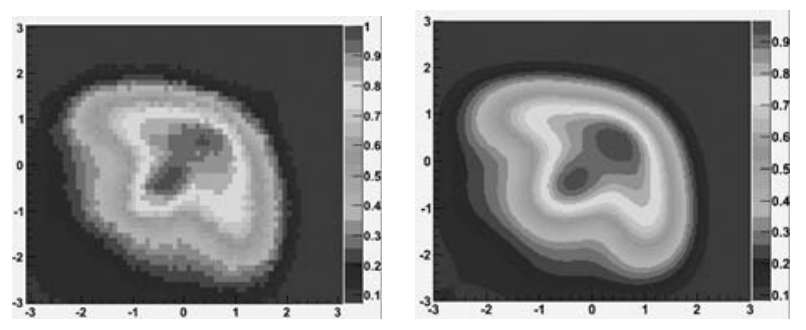

Fig. 4: PSFs for 800 pixels from the frame centre. Left: measured; Right: polynomial model
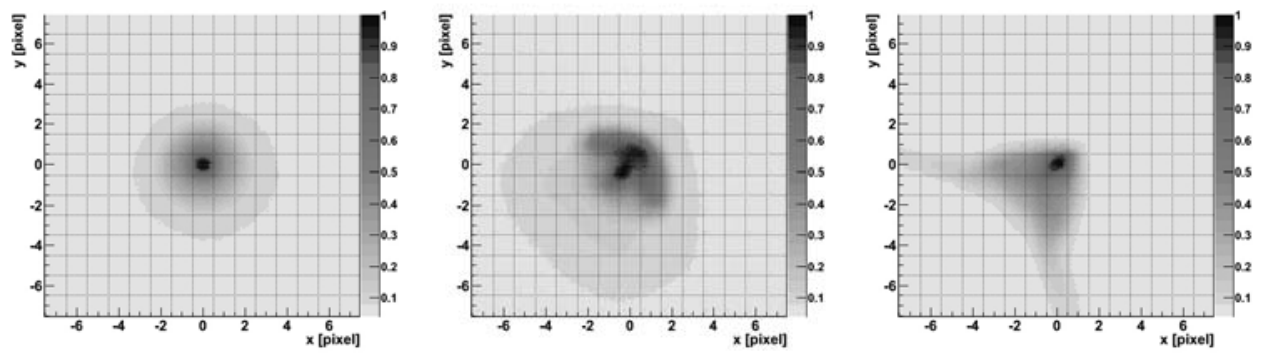

Fig. 3: Measured PSFs 0, 800 and 1400 pixels from the centre of the frame, along the diagonal 

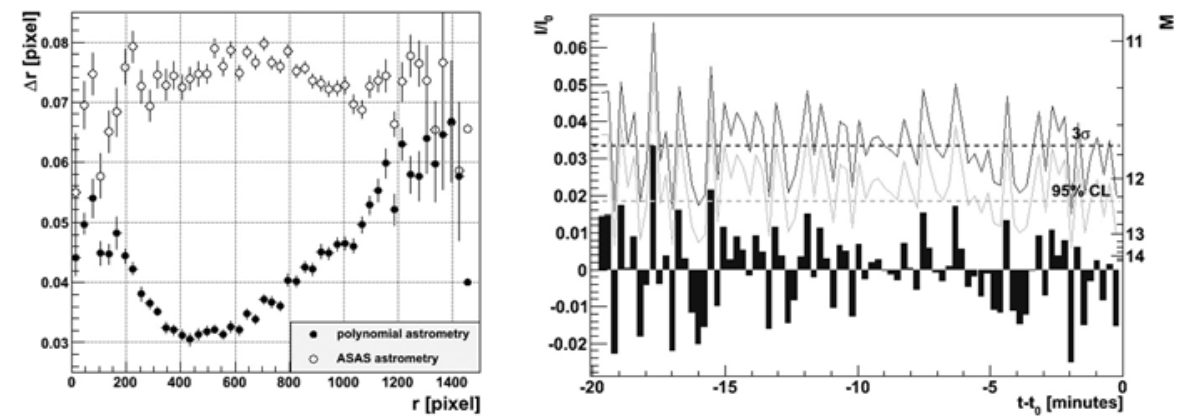

Fig. 5: Left: Comparison of the position determination accuracy from polynomial model profile astrometry and from ASAS aperture astrometry, for stars brighter than $9^{\mathrm{m}}$; Right: The signal resulting from the PSF model fit at the "nakedeye" burst position prior to the explosion (bars) and the extracted precursor limits (lines) (data from one camera)
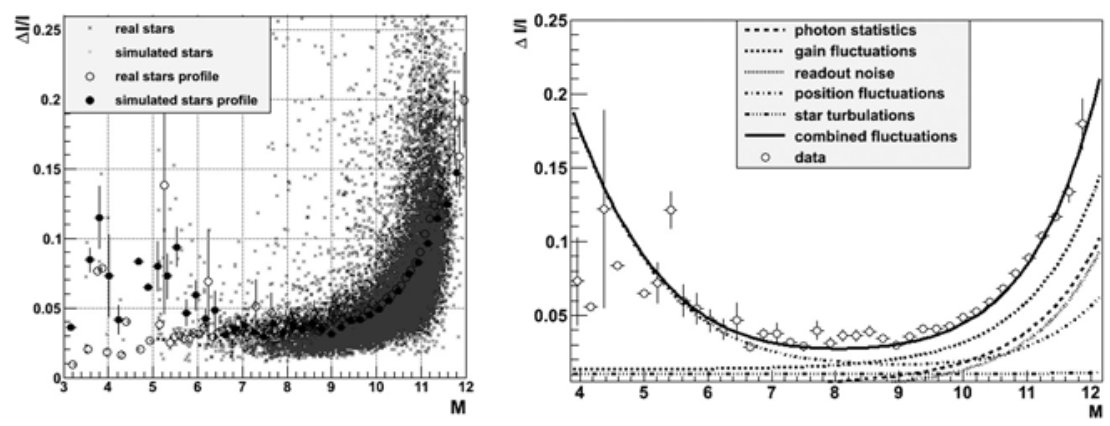

Fig. 6: Left: Polynomial photometry results for simulated and real sky images; Right: Relative error of the photometry performed on the simulated data, as a function of the star brightness (points) and the estimated contribution from different sources of variability (lines)

\section{Model applications}

The polynomial model that is obtained can be used for multiple purposes. The most straightforward use is for photometry and astrometry of stars, GRBs and other objects of interest. As for the photometry, when testing on selected data samples the model did not prove better in determining the brightness of stars than the ASAS aperture photometry used in our experiment. This is probably due to the real fluctuations of the star signal, caused by miscellaneous factors in the experiment that dominate the measurement. However, determining the position of the stars on the frame is up to a factor of 2.5 more precise in the polynomial PSF model (Figure 5, left).

Another application of the model is in a dedicated search for a signal in specific coordinates, such as a search for an optical precursor to the "naked-eye" GRB080319B [5]. Pi of the Sky started observing the position of the burst more than 19 minutes before the trigger, thus providing data well suited for this task. No signal exceeding $3 \sigma$, coinciding on two cameras, was found when fitting the modelled PSF to the frames preceding the burst, and the limiting magnitudo was improved from $11.5^{\mathrm{m}}$ (resulting from standard photometry) to $12.25^{\mathrm{m}}$ (Figure 5, right).
Additionally, a feature-rich simulator of the $\mathrm{Pi}$ of the Sky frame was created, utilizing the modelled detector response. The photometry results for real and simulated data are in very good agreement (Figure 6 , left). This can be taken as proof of good simulation quality. To obtain photometric uncertainties similar to the real uncertainties, fluctuations such as photon statistics, gain fluctuations, readout noise, position fluctuations and star turbulences had to be taken into account. The simulation results show that the photometry quality for stars darker then $8.5^{\mathrm{m}}$ is most probably limited by gain fluctuations, and not by readout noise, as had previously been anticipated (Figure 6, right).

\section{Summary}

Due to the very large field of view, PSF in the Pi of the Sky detector is highly deformed and is not described by general models. On the basis of dedicated laboratory measurements, we have created an effective model describing the dependence of PSF on the position of the star in the frame. These results may help in further understanding and developing the $\mathrm{Pi}$ of the Sky detector and other future wide field experiments. So far we have successfully introduced 
the model into a photometric algorithm, a dedicated signal search and astrometric algorithms, the last of which has given very promising results. Additionally, a detailed simulator of the $\mathrm{Pi}$ of the Sky frame has been created.

\section{Acknowledgement}

This work describes a research project supported by the Polish Ministry of Science and Higher Education in 2009-2011.

\section{References}

[1] Rerabek, M., Pata, P.: The space variant PSF for deconvolution of wide-field astronomical images, Proceedings of SPIE - 7015 - Adaptive Optics Systems. Washington : SPIE, 2008, p. 70152G-1-70152G-12.
[2] Siudek, M., et al.: Pi of the Sky telescopes in Spain and Chile, these proceedings.

[3] Racusin, J. L., et al.: Broadband observations of the naked-eye big $\gamma$-ray burst GRB 080319B, $\mathrm{Na}$ ture 455, 183-188, 2008.

[4] Toyozumi, H., Ashley, M. C. B.: Intra-pixel sensitivity variation and charge transfer inefficiency results of CCD scans, Publications of the Astronomical Society of Australia, 2005, 22, 257-266.

[5] Paczynski, B.: Optical Flashes Preceding GRBs, astro-ph/0108522, 2001.

Lech Wiktor Piotrowski

Aleksander Filip Żarnecki

Faculty of Physics

University of Warsaw

Hoża 69, 00-681 Warsaw 